\title{
Conservation Form of Helbing's Fluid Dynamic Traffic Flow Model *
}

\author{
Shu-feng Li(李书峰 $)^{1,3}$, Peng Zhang(张鹏 $)^{1,3}, \quad$ S.C. Wong(黄仕进 $)^{2}$ \\ (1. Shanghai Institute of Applied Mathematics and Mechanics, Shanghai University, \\ Shanghai, 200072, P.R. China) \\ (2. Department of Civil Engineering, The University of Hong Kong, Pokfulam Road, \\ Hong Kong SAR, P.R. China) \\ (3. Shanghai Key Laboratory of Mechanics in Energy Engineering)
}

\begin{abstract}
A standard conservation form is derived in this paper. The hyperbolicity of Helbing' s fluid dynamic traffic flow model is proved, which is essential to the general analytical and numerical study of this model. On the basis of this conservation form, a local discontinuous Galerkin scheme is designed to solve the resulting system efficiently. The evolution of an unstable equilibrium traffic state leading to a stable stop-and-go traveling wave is simulated. This simulation also verifies that the model is truly improved by the introduction of the modified diffusion coefficients, and thus helps to protect vehicles from collisions and avoide the appearance of the extremely large density.
\end{abstract}

Keywords. conservation form; hyperbolicity; local discontinuous Galerkin method; stopand-go wave.

\section{Introduction}

The fluid dynamic traffic flow model was first proposed independently by Lighthill and Whitham [1] and Richards [2], and has thus become known in the literature as the LWR model. In the theory underpinning the LWR model, traffic flow is viewed as a continuum that satisfies the following mass conservation.

$$
\frac{\partial \rho}{\partial t}+\frac{\partial \rho V}{\partial x}=0
$$

where $\rho(x, t)$ is the density, and $V(x, t)$ is the average velocity. To complete the equation, the LWR model also assumes an equilibrium velocity-density relationship, $V=V_{e}(\rho)$, which simply suggests a fixed curve in the velocity-density (or flow-density) phase plane. However, in many situations, this does not conform to the empirical data.

Hence, to improve the LWR model, many subsequent researchers have taken the traffic acceleration into account, and most of their formulations generally take the following form.

$$
\frac{\partial V}{\partial t}+V \frac{\partial V}{\partial x}=\frac{V_{e}(\rho)-V}{\tau}-\frac{1}{\rho} \frac{\partial P}{\partial x},
$$

where the first and second terms on the right-hand side are called relaxation and anticipation, respectively, with relaxation time $\tau$ and pressure $P$. The first term conveys a simple message: a vehicle accelerates if its current speed, $V$, is less than the equilibrium speed, $V_{e}(\rho)$; otherwise, it decelerates. Because the pressure, $P=P(\rho)$, is assumed to be an increasing function, with $P^{\prime}(\rho)>0$, the vehicle accelerates if the density in the downstream is decreasing with $\rho_{x}<0$; otherwise, it decelerates. Hence, whether the vehicle accelerates or decelerates depends on interaction between relaxation and pressure.

* The work that is described in this paper was jointly supported by grants from the National Natural Science Foundation of China (11072141), the Shanghai Program for Innovative Research Team in Universities, the University Research Committee of the University of Hong Kong (201007176059), and an Outstanding Researcher Award from the University of Hong Kong.

Corresponding author: Peng Zhang, Professor; E-mail: pzhang@mail.shu.edu.cn 
In the higher-order model first proposed by Payne [3] and Whitham [4] (the PW model), pressure $P=c_{0}^{2} \rho$, where the constant $c_{0}>0$ is the sonic speed. Kerner and Konhäuser [5] (KK) suggested that the pressure $P=c_{0}^{2} \rho-\eta_{0} V_{x}$, where the constant $\eta_{0}>0$ is the diffusion coefficient. Moreover, they significantly improved the PW model by adopting a non-convex fundamental diagram that is defined as the function $Q_{e}(\rho) \equiv \rho V_{e}(\rho)$. Through the relaxationpressure interaction, the model is able to reproduce the stop-and-go wave, which is the most important wave in traffic flow. See references [6-11] for other formulations or studies of the higher-order traffic flow model.

In an attempt to reduce the complexity of the Boltzmann-like traffic flow models in [12-16] (see also subsequent studies in [17-20]), and also based on the physics of gas kinetics, Helbing [21] proposed the following formulation for traffic pressure.

$$
P=\frac{\rho \Theta}{1-\rho s(V)}-\frac{\eta_{0}}{1-\rho s(V)} \frac{\partial V}{\partial x},
$$

where $\Theta(x, t)$ is the velocity variance, which is unknown and can be described by an equation analogous to thermal conduction:

$$
\frac{\partial \Theta}{\partial t}+V \frac{\partial \Theta}{\partial x}=-\frac{2 P}{\rho} \frac{\partial V}{\partial x}-\frac{1}{\rho} \frac{\partial J}{\partial x}+\frac{2}{\tau}\left(\Theta_{e}(\rho)-\Theta\right),
$$

with

$$
J=-\frac{\kappa_{0}}{1-\rho s(V)} \frac{\partial \Theta}{\partial x} .
$$

Here, $J$ describes the flux of velocity variance, $\Theta_{e}(\rho)$ is the desired or equilibrium state of velocity invariance, and $\kappa_{0}>0$ is constant. Similarly to Eq. (2), Eq. (4) includes such effects as convection, diffusion, and relaxation, and the system of (1), (2), and (4) constitutes a fluid dynamic model. Helbing [21] preliminarily set $s(V)=0$, by which we call the resulting system the original model. However, he considered that it was more reasonable to set

$$
s(V)=l+V \Delta T,
$$

where $l$ is the average vehicle length, $\Delta T$ is (the average of a driver's) reaction time, and $V \Delta T$ therefore denotes a safe distance. Based on Eq. (6), we call the resultant system the improved model. Helbing's formulation can also be viewed as an improvement of the KK model, to which the system is reduced by removing Eqs. (4) and (5) and setting $\Theta=c_{0}^{2}$ and $s(V)=0$ in Eq. (3).

The present paper defines a standard conservation form, and proves the hyperbolicity, of Helbing's fluid dynamic traffic flow model. Doing so is essential to validate the model by demonstrating finite propagation velocities, as is required for a description of traffic flow, and to ensure a certain definition of the weak solution. Although momentum and energy do not exist in traffic flow, a certain definition of conservation is mathematically required. Readers are referred to [8], in which two different conservation forms of the PW model are defined and shown to generate different solutions with different shock profiles. In this context, we note that, to date, neither the hyperbolicity nor any conservation form of Helbing's fluid dynamic model has yet been attained in the literature (see the discussion in Section 2).

The conservation form is more practical for the design of numerical schemes, as it is based on the weak solution theory of hyperbolic conservation laws [22]. The proper handling of the dissipation matrix allows the local discontinuous Galerkin (LDG) method [23-25] to be applied in solving the system. Although the system includes relaxation, which suggests complex coupling effects with convection and diffusion and for which some nonlinear numerical stabilities are unnecessarily ensured for the standard LDG method [23,24], the scheme is shown to be efficient in generating stable and convergent solutions for considerably small diffusion coefficients. These issues are discussed in Section 3, and we conclude the paper in Section 4. 


\section{Conservation form and fundamental properties of the model equations}

It is convenient to use dimensionless variables with the following scalings.

$$
\begin{aligned}
& x \rightarrow L x, t \rightarrow L t / V_{f}, \rho \rightarrow \rho_{j a m} \rho, V \rightarrow V_{f} V, V_{e}(\rho) \rightarrow V_{f} V_{e}(\rho), \Theta \rightarrow \Theta_{0} \Theta, \\
& \Theta_{e}(\rho) \rightarrow \Theta_{0} \Theta_{e}(\rho), \eta_{0} \rightarrow L \rho_{j a m} V_{f} \eta_{0}, \kappa_{0} \rightarrow L \rho_{j a m} V_{f} \kappa_{0}, \tau \rightarrow L \tau / V_{f},
\end{aligned}
$$

where $L$ is the length of the computational interval $[0, L], V_{f}$ is the free-flow velocity, $\rho_{j a m}$ $\left(\equiv l^{-1}\right)$ is the maximal density, and $\Theta_{0}$ is the maximal velocity variance, which, together with functions $V_{e}(\rho)$ and $\Theta_{e}(\rho)$, are given in Section 2. Moreover, we define $c_{0}=\sqrt{\Theta_{0}} / V_{f}$ and $s_{0}=\rho_{\text {jam }} V_{f} \Delta T$. Despite possible changes in their forms, the resultant equations of (1)-(6) are still referred to in the following operations by the same serial numbers correspondingly.

\subsection{Conservation of the system}

Equation (1) refers to mass conservation. As is well known in fluid dynamics, the combination of $(1) \times V+(2) \times \rho$ leads to

$$
\frac{\partial(\rho V)}{\partial t}+\frac{\partial\left(\rho V^{2}+P\right)}{\partial x}=\frac{\rho}{\tau}\left(V_{e}(\rho)-V\right)
$$

which is similar to the conservation of momentum. However, it seems appropriate to proceed to the combination of $(1) \times\left(V^{2}+c_{0}^{2} \Theta\right)+(2) \times 2 \rho V+(4) \times \rho$, which yields

$$
\frac{\partial\left(\rho V^{2}+c_{0}^{2} \rho \Theta\right)}{\partial t}+\frac{\partial\left(\rho V^{3}+c_{0}^{2} \rho V \Theta+2 P V\right)}{\partial x}=\frac{2 \rho V\left(V_{e}(\rho)-V\right)}{\tau}-\frac{\partial J}{\partial x}+\frac{2 c_{0}^{2} \rho}{\tau}\left(\Theta_{e}(\rho)-\Theta\right) .
$$

Equation (8) is analogous to the conservation of energy. We now substitute Eqs. (3), (5), and (6) into Eqs. (7) and (8); then, Eqs. (1), (7), and (8) give the following conservative system.

$$
\left\{\begin{array}{l}
\frac{\partial \rho}{\partial t}+\frac{\partial(\rho V)}{\partial x}=0, \\
\frac{\partial(\rho V)}{\partial t}+\frac{\partial\left(\rho V^{2}+\frac{c_{0}^{2} \rho \Theta}{1-\rho s(V)}\right)}{\partial x}=\frac{\rho}{\tau}\left(V_{e}(\rho)-V\right)+\frac{\partial}{\partial x}\left(\frac{\eta_{0}}{1-\rho s(V)} \frac{\partial V}{\partial x}\right) \\
\frac{\partial\left(\rho V^{2}+c_{0}^{2} \rho \Theta\right)}{\partial t}+\frac{\partial\left(\rho V^{3}+c_{0}^{2} \rho V \Theta+\frac{2 c_{0}^{2} \rho V \Theta}{1-\rho s(V)}\right)}{\partial x}=\frac{2 \rho V}{\tau}\left(V_{e}(\rho)-V\right) \\
+\frac{2 c_{0}^{2} \rho}{\tau}\left(\Theta_{e}(\rho)-\Theta\right)+\frac{\partial}{\partial x}\left(\frac{2 \eta_{0} V}{1-\rho s(V)} \frac{\partial V}{\partial x}\right)+\frac{\partial}{\partial x}\left(\frac{c_{0}^{2} \kappa_{0}}{1-\rho s(V)} \frac{\partial \Theta}{\partial x}\right)
\end{array}\right.
$$

System (9) may be analogous to the Navier-Stokes equations in fluid dynamics, although we still need to show clearly that the convection component of the system is hyperbolic.

\subsection{Hyperbolicity of the system}

Denote the conservative variables by $\mathbf{u}=\left(u_{1}, u_{2}, u_{3}\right)^{\mathbf{T}}=\left(\rho, \rho V, \rho V^{2}+c_{0}^{2} \rho \Theta\right)^{\mathbf{T}}$. We rewrite system (9) in the following vector form.

$$
\mathbf{u}_{t}+\mathbf{f}(\mathbf{u})_{x}=\mathbf{S}(\mathbf{u})+\left(\epsilon(\mathbf{u}) \mathbf{u}_{x}\right)_{x},
$$

with flux vector

$$
\mathbf{f}(\mathbf{u})=\left(f_{1}(\mathbf{u}), f_{2}(\mathbf{u}), f_{3}(\mathbf{u})\right)^{\mathbf{T}}=\left(u_{2}, \frac{u_{2}^{2}}{u_{1}}+\frac{u_{1} u_{3}-u_{2}^{2}}{u_{1}\left(1-u_{1}-s_{0} u_{2}\right)}, \frac{u_{2} u_{3}}{u_{1}}+\frac{2\left(u_{1} u_{2} u_{3}-u_{2}^{3}\right)}{u_{1}^{2}\left(1-u_{1}-s_{0} u_{2}\right)}\right)^{\mathbf{T}},
$$

source vector

$$
\mathbf{S}(\mathbf{u})=\left(0, \frac{u_{1} V_{e}\left(u_{1}\right)-u_{2}}{\tau}, \frac{2\left[u_{2} V_{e}\left(u_{1}\right)+c_{0}^{2} u_{1} \Theta_{e}\left(u_{1}\right)-u_{3}\right]}{\tau}\right)^{\mathbf{T}},
$$


and diffusion matrix

$$
\epsilon(\mathbf{u})=\left(\begin{array}{ccc}
0 & 0 & 0 \\
\frac{-\eta_{0} u_{2}}{u_{1}^{2}\left(1-u_{1}-s_{0} u_{2}\right)} & \frac{\eta_{0}}{u_{1}\left(1-u_{1}-s_{0} u_{2}\right)} & 0 \\
\frac{-\kappa_{0} u_{1} u_{3}+2\left(\kappa_{0}-\eta_{0}\right) u_{2}^{2}}{u_{1}^{3}\left(1-u_{1}-s_{0} u_{2}\right)} & \frac{2\left(\eta_{0}-\kappa_{0}\right) u_{2}}{u_{1}^{2}\left(1-u_{1}-s_{0} u_{2}\right)} & \frac{\kappa_{0}}{u_{1}\left(1-u_{1}-s_{0} u_{2}\right)}
\end{array}\right),
$$

which is standard when referring to the hyperbolic conservation law with diffusion [22].

Solving the eigenpolynomial $|\partial \mathbf{f}(\mathbf{u}) / \partial \mathbf{u}-\lambda I|=0$, where $\partial \mathbf{f}(\mathbf{u}) / \partial \mathbf{u}$ is the Jacobian of $\mathbf{f}(\mathbf{u})$, and $I$ is the unit matrix, we derive the following eigenvalues.

$$
\left\{\begin{array}{l}
\lambda_{1}(\rho, V, \Theta)=V+\frac{s_{0} c_{0}^{2} \rho \Theta-\sqrt{\left(s_{0} c_{0}^{2} \rho \Theta\right)^{2}+12 c_{0}^{2} \Theta(1-\rho s(V))^{2}}}{2(1-\rho s(V))^{2}} \\
\lambda_{2}(\rho, V, \Theta)=V \\
\lambda_{3}(\rho, V, \Theta)=V+\frac{s_{0} c_{0}^{2} \rho \Theta+\sqrt{\left(s_{0} c_{0}^{2} \rho \Theta\right)^{2}+12 c_{0}^{2} \Theta(1-\rho s(V))^{2}}}{2(1-\rho s(V))^{2}}
\end{array}\right.
$$

Because $\left\{\lambda_{i}\right\}_{i=1}^{3}$ are distinct, $\lambda_{1}<\lambda_{2}<\lambda_{3}$, system (9) is strictly hyperbolic.

\section{Numerical implementation}

Only on the basis of the standard conservation form of (10) can appropriate numerical schemes be designed to resolve weak solutions of the model. With proper handling of the diffusion term, the LDG method is a good choice for achieving stable and convergent solutions that are physically relevant. The insight here is that diffusion should be incorporated into convection, such that the system appears to incorporate standard conservation laws, and convection and diffusion can be sufficiently well-balanced to achieve upwind effects. See references [23-25] for a more detailed discussion.

We also incorporate the relaxation source term into convection in the model discussed here, which may help to coordinate the interaction between all of the terms involved in a similar fashion.

\subsection{Numerical scheme for the system}

We introduce two new vectors: $\mathbf{q}=\mathbf{u}_{x}$ and $\mathbf{p}=\int_{0}^{x} \mathbf{S}(\mathbf{u}(\xi, t)) d \xi$. Thus, system (10) can be rewritten as

$$
\left\{\begin{array}{l}
\mathbf{u}_{t}+\mathbf{F}(\mathbf{u}, \mathbf{q}, \mathbf{p})_{x}=0 \\
\mathbf{q}-\mathbf{u}_{x}=0
\end{array}\right.
$$

where

$$
\mathbf{F}(\mathbf{u}, \mathbf{q}, \mathbf{p})=\mathbf{f}(\mathbf{u})-\mathbf{p}-\epsilon(\mathbf{u}) \mathbf{q} .
$$

Given initial conditions $\mathbf{u}(x, 0)=\mathbf{u}_{0}(x)$, and applying proper boundary conditions, the numerical solution $\mathbf{u}_{h}(x, t)$ of system (11) can be derived through the LDG method. The procedure can be summarized as follows.

1. For cell division $I_{j}=\left[x_{j-1 / 2}, x_{j+1 / 2}\right]$ of computational interval $[0, L]$ (or $[0,1]$ by scaling), approximate $\mathbf{u}(x, t)$ on $I_{j}$ by $\mathbf{u}_{h}(x, t) \in \mathbf{u}\left(I_{j}\right)$, where $\mathbf{u}\left(I_{j}\right)$ represents a polynomial space of, at most, degree $k$ on $I_{j}$.

2. Proceed to obtain the weak formation of (11) (and initial conditions $\mathbf{u}(x, 0)=\mathbf{u}_{0}(x)$ ) on $I_{j}$, which gives a semi-discrete scheme that can be denoted by the following ordinary differential equations (ODEs).

$$
\frac{d \mathbf{u}_{h}}{d t}=L\left(\mathbf{u}_{h}\right), x \in I_{j}
$$

3. Apply TVD Runge-Kutta time discretization to solve the ODEs of (12). 
We note that the cell boundary values of $\mathbf{F}\left(\mathbf{u}\left(x_{j+1 / 2}, t\right), \mathbf{q}\left(x_{j+1 / 2}, t\right), \mathbf{p}\left(x_{j+1 / 2}, t\right)\right)$, and $\mathbf{u}\left(x_{j+1 / 2}, t\right)$ in Step 2 are approximated by numerical fluxes $\hat{\mathbf{F}}$ and $\hat{\mathbf{u}}$. For the model under discussion here, these numerical flux functions are given by

$$
\begin{gathered}
\hat{\mathbf{F}}\left(\mathbf{u}^{-}, \mathbf{u}^{+} ; \mathbf{q}^{-}, \mathbf{q}^{+} ; \mathbf{p}\right)=\hat{\mathbf{f}}\left(\mathbf{u}^{-}, \mathbf{u}^{+}\right)-\frac{\epsilon\left(\mathbf{u}^{-}\right) \mathbf{q}^{-}+\epsilon\left(\mathbf{u}^{+}\right) \mathbf{q}^{+}}{2}-\mathbf{p}, \\
\hat{\mathbf{u}}\left(\mathbf{u}^{-}, \mathbf{u}^{+}\right)=\frac{\mathbf{u}^{-}+\mathbf{u}^{+}}{2},
\end{gathered}
$$

and $\hat{\mathbf{f}}\left(\mathbf{u}^{-}, \mathbf{u}^{+}\right)$is taken to be the Lax-Friedrichs numerical flux:

$$
\hat{\mathbf{f}}\left(\mathbf{u}^{-}, \mathbf{u}^{+}\right)=\frac{1}{2}\left[\mathbf{f}\left(\mathbf{u}^{-}\right)+\mathbf{f}\left(\mathbf{u}^{+}\right)-\alpha\left(\mathbf{u}^{+}-\mathbf{u}^{-}\right)\right], \alpha=\max _{\mathbf{u}} \max _{i \in\{1,2,3\}}\left|\lambda_{i}(\mathbf{u})\right| .
$$

Here, superscripts "-" and "+" denote the discontinuous values of the vectors on the left- and right-hand sides, respectively, of cell boundary $x=x_{j+1 / 2}$. Moreover, a slope limiter is applied to $\mathbf{u}_{h}$ for each iteration in Step 3. We choose $k=1$ in Steps 1 and 2, and apply third-order accurate time discretization in Step 3.

\subsection{Numerical simulation}

The parameters are given as

$$
\tau=0.5 \mathrm{~min}, \eta_{0}=600 \mathrm{~km} / \mathrm{h}, \kappa_{0}=600 \mathrm{~km} / \mathrm{h}, l=5 \mathrm{~m}, \Delta T=0.75 \mathrm{~s}, L=10 \mathrm{~km} .
$$

Functions $V_{e}(\rho)$ and $\Theta_{e}(\rho)$ in Eqs. (2)-(5) are given by

$$
\begin{aligned}
& V_{e}(\rho)=V_{f}\left\{\left[1+\exp \left(\frac{\rho / \rho_{\text {jam }}-0.25}{0.06}\right)\right]^{-1}-3.72 \times 10^{-6}\right\}, \\
& \Theta_{e}(\rho)=\Theta_{0}\left\{\left[1+\exp \left(\frac{\rho / \rho_{\text {jam }}-0.25}{0.06}\right)\right]^{-1}-3.72 \times 10^{-6}\right\},
\end{aligned}
$$

with $V_{f}=120 \mathrm{~km} / \mathrm{h}, \rho_{\text {jam }}=200 / \mathrm{km}$, and $\Theta_{0}=(45 \mathrm{~km} / \mathrm{h})^{2}$. We apply the periodic boundary conditions and assume the following initial conditions.

$$
\left\{\begin{array}{l}
\rho(x, 0)=0.3 \rho_{\text {jam }}, \\
V(x, 0)=V_{e}(\rho(x, 0))(1+0.01 \sin (2 \pi x / L)), \\
\Theta(x, 0)=\Theta_{e}(\rho(x, 0)) .
\end{array}\right.
$$

All of these settings are the same as those in Helbing [21].

The initial values of (13) obviously suggest an equilibrium solution to the model if the term $0.01 \sin (2 \pi x / L)$ is removed. In other words, this term serves as a small perturbation to the equilibrium solution. Because linear stability analysis of both the original and improved models shows the equilibrium solution to be unstable [21], traffic flow is expected to be far from the equilibrium state. Figure 1 shows the evolution of density for $t \leq 1 \mathrm{~h}$. Figure 2 shows that traffic flow eventually evolves into a stable profile that closely resemble the traveling wave known as the stop-and-go wave in the literature.

Comparison of these figures indicate that the improved model has a smaller maximal density and smoother profile than the original model. This is due to the difference between the diffusion coefficients in the conservation form of (9), which physics requires to be non-negative to ensure the stability of the model. This requirement is self-evident for the original model. However, for the improved model, it implies that

$$
1-\rho s(V) \geq 0, \text { or } \rho \leq \frac{\rho_{\text {jam }}}{1+V \Delta T \rho_{\text {jam }}},
$$




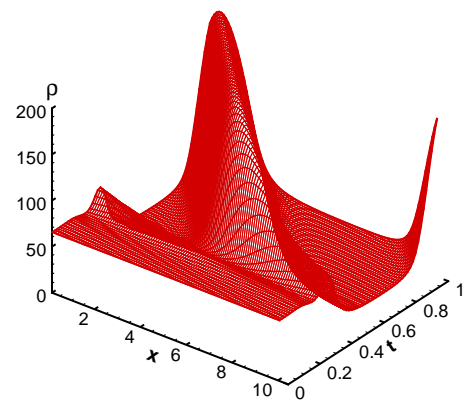

(a)

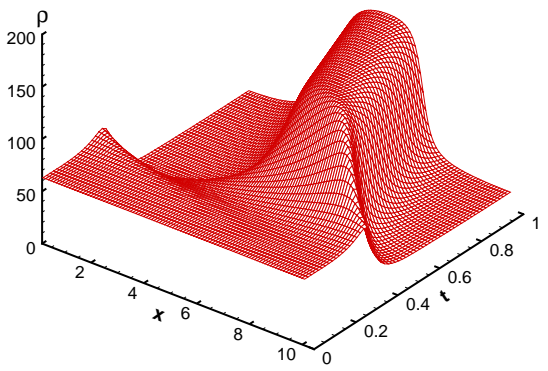

(b)

Fig. 1 Evolution of traffic flow under the perturbed equilibrium initial state of (13): (a) with $s(V)=$ 0 , which corresponds to the original model; and (b) with $s(V)$ given by (6), which corresponds to the improved model.

(a)

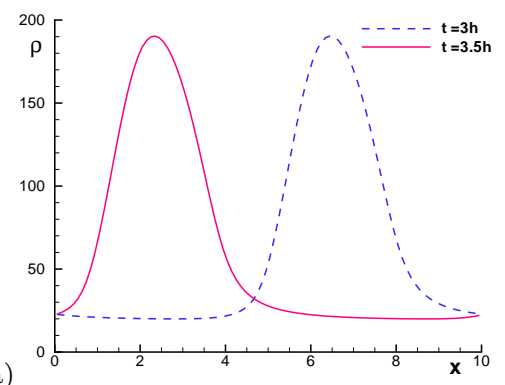

(b)

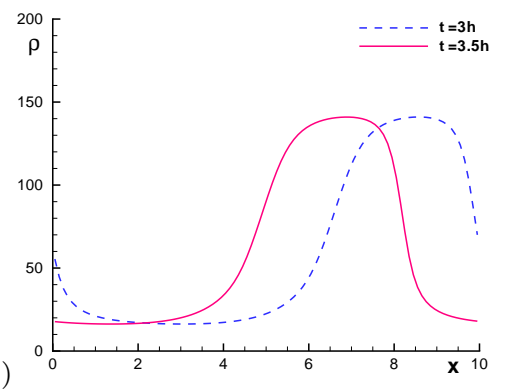

Fig. 2 Density profiles indicating a stop-and-go traveling wave, simulated by (a) the original model and (b) the improved model.

which sets an upper bound that is smaller than $\rho_{\text {jam }}$. By the inequality, it is also obvious that the earlier a driver reacts to a change in the traffic flow ahead, the smaller the upper bound. In this regard, the model is truly improved, as it avoids the appearance of extremely large density and traffic collisions.

Figure 1(b) exhibits almost the same solution profile as that given by Helbing [21], who adopted the same parameters, but based his model on the nonconservative system and applied the classical Lax-Wendroff (LW) scheme. We believe the coincidence to be due to the large coefficients $\eta_{0}=\kappa_{0}=600 \mathrm{~km} / \mathrm{h}$, which represent the speed of the diffusions. It is well known that a large diffusion helps to improve numerical stability of classical methods such as the LW scheme. Moreover, large diffusion suggests a rather smooth solution profile, which greatly reduces the difference between the numerical solutions generated by the nonconservative and conservative systems. It is also well known that as the diffusion $\left(\eta_{0}\right.$ and $\left.\kappa_{0}\right)$ decreases, a classical finite difference scheme such as the LW scheme will generate non-physical oscillations or lose its stability completely.

We note that $\eta_{0}$ and $\kappa_{0}$ are theoretical parameters, which should allow an extensive choice of values for comparison with the observed data. The numerical scheme should thus accommodate the variability of $\eta_{0}$ and $\kappa_{0}$, particularly for smaller values. The LDG scheme ensures some nonlinear (e.g., $L_{1}$ and TV) stabilities for standard convection-diffusion systems with sufficiently small diffusion coefficients [23,24]. However, similar stabilities are not theoretically guaranteed for the system under discussion here, which also includes relaxation source terms. 
(a)

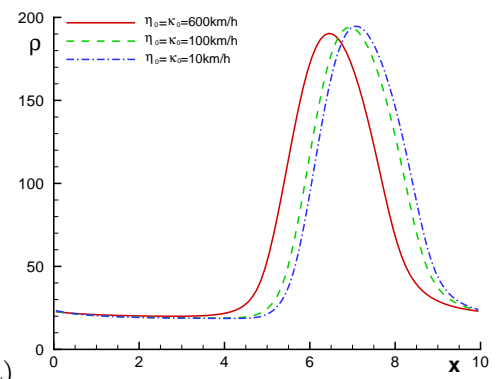

(b)

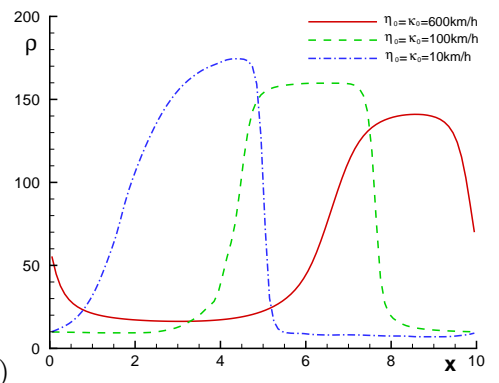

Fig. 3 Density profiles at $t=3 h$, with smaller diffusion coefficients $\eta_{0}=\kappa_{0}$ and simulated by (a) the original model and (b) the improved model.

By setting the other parameters to be the same as those in Figures 1 and 2, we obtain the numerical results for the three smaller $\eta_{0}=\kappa_{0}$, as shown in Figure 3. For both the original and improved models, as the diffusion decreases, we see a reasonable increase in the maximal density. At the same time, the downstream front of the stop-and-go wave becomes steeper, and is very close to a shock for the improved model, whereas the smoothness of this front changes little for the original model.

Being sensitive to diffusion and moving backward, this front (particularly for the improved model) is associated primarily with the $\lambda_{1}$-characteristic field, of which the sonic speed, $c_{1} \equiv$ $V-\lambda_{1}$, is an increasing function of $s(V)$. We note that a larger sonic speed implies stronger nonlinearity. Therefore, this characteristic field suggests stronger nonlinearity for the improved model (with $s(V)>0$ ) than for the original model (with $s(V)=0$ ), which explains the difference in the changes for the downstream front in Figure 3(a) and (b).

The choice of $\eta_{0}=\kappa_{0}=10 \mathrm{~km} / \mathrm{h}$ was made because it is sufficiently small for comparison with the observed data. However, if we set $\eta_{0}=\kappa_{0}=5 \mathrm{~km} / \mathrm{h}$ or even smaller to test for numerical stability, then we derive distorted or unstable solution profiles. A suggested improvement is to include an "upwind" limiter that can also incorporate relaxation into convection and diffusion, and it should be based on the system of (11).

\section{Conclusion}

We derive the standard conservation form, and prove the hyperbolicity, of Helbing's dynamic traffic flow model. We show that with the proper handling of the diffusion and relaxation terms, a LDG scheme can be designed to solve the model. The numerical simulation reproduces the stop-and-go wave that evolves from the unstable equilibrium traffic state. In comparison with the original model, simulation of the improved model generates a smoother profile and a smaller maximal density for the stop-and-go traveling wave. From the diffusion coefficients, we conclude that the improved model suggests a smaller upper bound of density, which also implies that the formulation is collision-free. Drawing on the conservation form and the scheme designed herein, additional properties of the model may be discovered in future research.

The LDG scheme applied here ensures stable numerical solutions to the model for very small diffusion coefficients. However, the scheme could be further improved by allowing a sufficiently small degree of diffusion.

\section{References}

[1] Lighthill, M.J. and Whitham, G.B. On kinematic waves II: A theory of traffic flow on long crowded roads, Proc. Roy. Soc. A, 229, 317-345 (1955).

[2] Richards, P.I. Shock waves on the highway. Operations Research, 4 42-51 (1956). 
[3] Payne, H.J. Models of freeway traffic and control, In: A.G. Bekey (ed.), Mathematical Models of Public Systems, Simulation Council Proc., La Jolla, 1, 51-61 (1971).

[4] Whitham, G.B. Linear and Nonlinear Waves, John Wiley and Sons, New York (1974).

[5] Kerner, B.S. and Konhäuser, P. Structure and parameters of clusters in traffic flow. Phys. Rev. E, 50, 54-83 (1994).

[6] Siebel, F. and Mauser, W. On the fundamental diagram of traffic flow, SIAM J. Appl. Math., 66, 1150-1162 (2006).

[7] Zhang, P., Wong, S.C. and Dai, S.Q. Characteristic parameters of a wide cluster in a higher-order traffic flow model, Chin. Phys. Lett., 23, 516-519 (2006).

[8] Zhang, P. and Wong, S.C. Essence of conservation forms in the traveling wave solutions of higherorder traffic flow models. Phys. Rev. E, 74(2), 026109 (2006).

[9] Xu, R.Y., Zhang, P., Dai, S.Q. and Wong, S.C. Admissibility of a wide cluster solution in anisotropic higher-order traffic flow models. SIAM J. Appl. Math., 68(2), 562-573 (2007).

[10] Zhang, P., Wong S.C. and Dai, S.Q. A conserved higher-order anisotropic traffic flow model: Description of equilibrium and non-equilibrium flows, Transpn. Res. Part B, 43, 562-574 (2009).

[11] Tang, T.Q., Huang, H.J. and Shang, H.Y. A new macro model for traffic flow with the consideration of the driver's forecast effect, Physics Letters A, 374, 1668-1672 (2010).

[12] Prigogine, I. and Herman, R. Kinetic Theory of Vehicular Traffic, American Elsevier Publishing Co., New York (1971).

[13] Paveri-Fontana, S.L. On Boltzmann-like treatments for traffic flow: A critical review of the basic model and an alternative proposal for dilute traffic analysis, Transpn. Res., 9(4), 225-235 (1975).

[14] Phillips, W. Kinetic Model for Traffic Flow, National Technical Information Service, Springfield, VA (1977).

[15] Helbing, D. A fluid-dynamic model for the movement of pedestrians, Complex Systems, 6, 391-415 (1992).

[16] Helbing, D. Hennecke, A. Shvetsov, V. and Treiber, M. Master: macroscopic traffic simulation based on a gas-kinetic, non-local traffic model. Transpn. Res. Part B, 35, 183-211 (2001).

[17] Hoogendoorn, S.P. and Bovy, P.H.L. Continuum modeling of multiclass traffic flow. Transpn. Res. Part B, 34, 123-146 (2000).

[18] Hoogendoorn, S.P. and Bovy, P.H.L. Generic gas-kinetic traffic systems modeling with appications to vehicular traffic flow. Transpn. Res. Part B, 35, 317-336 (2001).

[19] Ngoduy, D. Derivation of continuum traffic model for weaving sections on freeways. Transportmetrica, 2, 199-222 (2006).

[20] Ngoduy, D. Application of gas-kinetic theory to modeling mixed traffic of manual and ACC vehicles. Transportmetrica, (DOI: 10.1080/18128600903578843).

[21] Helbing, D. Improved fluid-dynamic model for vehicular traffic. Phys. Rev. E, 51(4), 3164-3169 (1995).

[22] Liu, T.P. Hyperbolic and viscous conservation laws, CBMS-NSF Regional Conference Series in Applied Mathematics, 72, SIAM, Philadelphia, PA (2000).

[23] Cockburn, B., Lin S.Y. and Shu, C.-W. TVB Runge-Kutta local projection discontinuous Galerkin finite element method for conservation laws III: One dimensional systems. J. Comput. Phys., 84, 90-113 (1989).

[24] Cockburn, B. and Shu, C.-W. The local discontinuous Galerkin method for time-dependent convection-diffusion systems. SIAM J. Numer. Anal., 35, 2440-2463 (1998).

[25] Bassi, F. and Rebay, S. A higher-order accurate discontinuous finite element method for the numerical solution of the compressible Navier-Stokes equations. J. Comput. Phys., 131, 267-279 (1997). 